\title{
Bounded modes to the rescue of optical transmission [ool 10.105/1/Rer2007011]
}

\author{
Michaël Sarrazin and Jean-Pol Vigneron, \\ Laboratoire de Physique du Solide, Département de Physique • FUNDP • 61 rue de Bruxelles • B-5000 Namur • Belgium \\ E-mail : michael.sarrazin@fundp.ac.be
}

$I^{n}$ $\mathrm{n}$ the beginning of the $19^{\text {th }}$ century, T. Young and J. Fraunhofer built the first optical diffraction gratings and showed the role of optical diffraction in their behaviour. Since that time, diffraction gratings have been used in a broad range of technological applications, from the earlier accurate optical spectrometers to the recent integrated optical devices. They are also used in a broad range of wavelength from X-ray to microwaves. In this context, diffraction gratings have been the cornerstones of many publications for almost two centuries. Surprisingly, nowadays the subject is far from being exhausted.

Every student knows that a grating can spread a white light beam from an incandescent lamp into a continuous spectrum of colors. For a grating made with a one-dimensional lattice of thickthin wires, this "rainbow" is duplicated many times, each one corresponding to a diffraction order $m$ (See Fig.1 for instance). These basic behaviours of diffraction gratings can be easily understood by the conservation of light momentum such as

$$
\vec{k}_{s}=\vec{k}_{i}+\vec{K}
$$

where $\vec{k}_{s}$ is the momentum of the scattered light, $\vec{k}_{i}$ is the momentum of the incident light, and $\vec{K}$ is a vector of the reciprocal lattice of the grating such that $\vec{K} \cdot \vec{a}=2 \pi m(m \in \wedge)$, where $\vec{a}$ is the basis vector of the lattice. The general behaviour of optical grating results from this basic assumption. For instance, in Fig.1, one considers a reflecting grating. An incident light $i$ is then scattered into three diffraction orders. The zero order $(m=0)$ is known as the specular beam. In Fig.1, two other orders are considered, $m=1$ and $m=-1$.

\section{Wood anomalies}

In 1902, R. W. Wood observed some unexpected patterns in the spectrum of light resolved by optical diffraction gratings [1]. The Wood spectrum presented many unusual rapid variations of its intensity in certain narrow wavelengths bands. As a consequence, these effects unexplained by ordinary grating theory were named "Wood anomalies".

In 1907, Lord Rayleigh proposed an explanation of those anomalies [2]. Considering for instance the case of the Fig.1, one notes that the order $m=1$ becomes tangent to the surface grating before disappearing. In such a case, the light momentum component along the $\mathrm{O} z$ axis of a diffracted order $m$, i.e. $k_{z, m}$, becomes imaginary right after having been cancelled. Then, the diffracted order becomes evanescent (non-homogeneous order). When $k_{z, m}$ is real, the diffracted order is free to propagate along the $\mathrm{O} z$ axis (homogeneous order). In the present case, as the order $m=1$ turns to be non-homogeneous, its energy will be redistributed over the other orders. As a conse- quence, the diffracted beam intensity of the specular beam $(m=0)$, for instance, increases just as the diffracted order $m=1$ vanishes. For an incidence $\theta$ (see Fig.1), a diffracted order $m$ becomes non-homogeneous for wavelengths greater than a specific value, the Rayleigh wavelength, for which an anomaly occurs. One then talks about a Rayleigh anomaly.

Around 1938, U. Fano proposed another explanation [3], where the anomalies are related to a resonance effect. Such a resonance comes from a coupling between an eigenmode of the grating and a non-homogeneous diffraction order (one also talks about a resonant diffraction order), i.e. after it vanishes for wavelengths greater than the corresponding Rayleigh wavelength.

From an experimental point of view, gratings can then show some very complex behaviour often hard to explain in details without the use of Maxwell's equations. In addition, solving Maxwell's equations in order to study the electromagnetic diffraction quickly becomes a difficult problem, even with simple geometries. Except for some simple and powerful analytical works, such as those from Lord Rayleigh or U. Fano, it will be necessary to wait until the Sixties and the beginning of computer calculation to observe significant progress in the Wood anomalies comprehension. For instance, one can underline the first fundamental numerical results of Hessel and Oliner in 1965 [4], and Maystre and Nevière in 1977 [5,6]. Hessel and Oliner presented a wide study of the eigenmode resonance role in Wood anomalies [4]. They have shown that depending on the type of periodic structure, the two kinds of anomalies i.e., Rayleigh anomalies or resonant anomalies may occur separately or are almost superimposed.

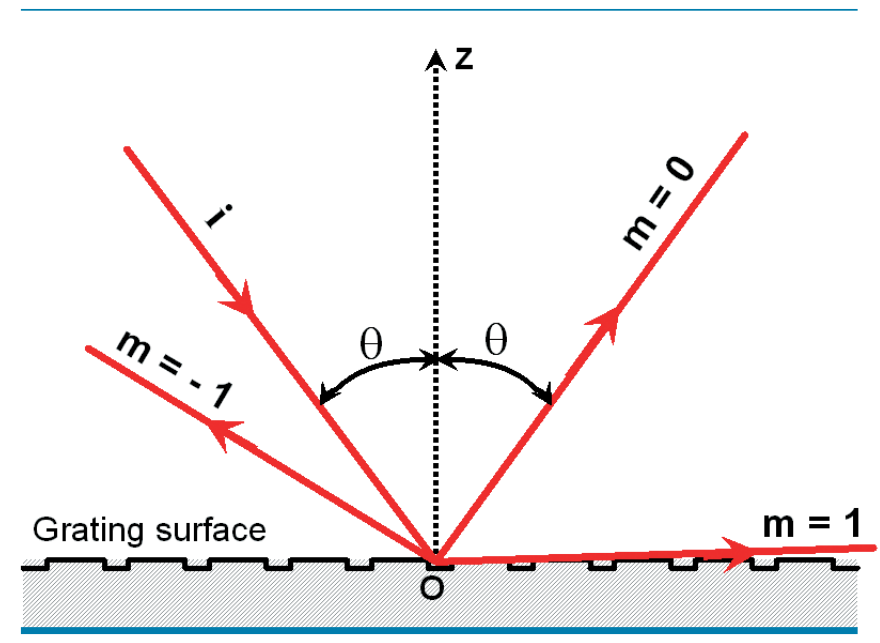

$\Delta$ Fig. 1: A reflecting optical grating. $i$ is the incident light beam. The light beams labelled $m$ are some of the diffracted orders and $\boldsymbol{\theta}$ is the angle of incidence. 
Maystre and Nevière studied specific cases of such resonant anomalies $[5,6]$. For instance, they presented a wide study about "plasmon anomalies", which occurs when the surface plasmons of a metallic grating are excited [5]. They also considered an anomaly that appears when a dielectric coating is deposited on a metallic grating, and corresponds to guided modes resonances in the dielectric layer [6].

It is important to underline that, in some recent publications, some authors improperly reduce the Wood anomalies to the Rayleigh anomalies only. It is important to recall that the Wood anomaly concept covers the resonant anomalies (Fano anomalies) and the Rayleigh anomalies at the same time. In addition, the resonant part of the phenomenon can imply many kinds of resonances and not only the surface plasmons resonances.

Amazingly, thirty years after the pioneer works, in the early $21^{\text {st }}$ century, the experimental and theoretical interest in these subjects has not dried up.

\section{Optical transmission in a nanostructured slab}

In 1998, Ebbesen et al [7] reported on optical transmission experiments performed on periodic arrays of subwavelength cylindrical holes drilled in a thin metallic layer deposited on glass (see Fig.2). These experiments renewed the motivation for investigating metallic gratings, in particular those with a two-dimensional lattice. The most attractive characteristic of their results was the peculiar wavelength dependence of the transmission (see Fig.3). The latter was defined as the ratio between the energy of the specular transmitted beam $(m=0)$ only and the energy of the incident beam. After these first experimental observations, the role of the thin metallic film surface plasmons (SPs) was put forward in order to explain the peculiar wavelength dependence of the transmission [7].

A surface plasmon on a plane surface is a non-radiative electromagnetic mode, associated with a collective excitation of electrons at the interface between a conductor and an insulator. In this way, a surface plasmon cannot be excited directly by light, and it cannot decay spontaneously into photons. The non-radiative nature of SPs is due to the fact that interaction between light and SPs cannot simultaneously satisfy energy and momentum conservation laws. Moreover, the momentum conservation requirement can be achieved by roughening or corrugating

Thin optical absorbing slab

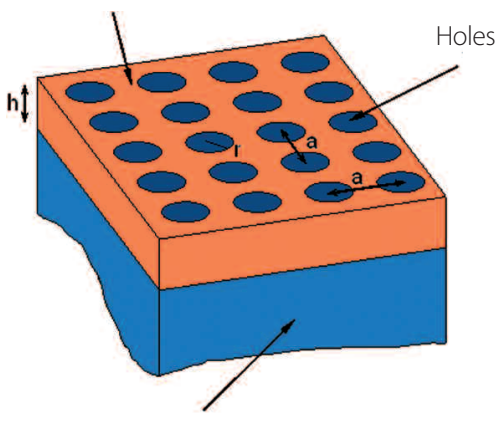

Dielectric substrate (with an imaginary part of the permittivity almost equal to zero the metal surface, for instance. It is exactly the situation in those devices. In this case, the transmission peaks were interpreted as SPs resonances [7]. Surprisingly, this approach seems to have been suggested independently of the knowledge of some

4 Fig. 2: The typical bidimensional array of subwavelength holes. earlier works about the reflecting one-dimensional gratings. Then, the first attempt to understand the Ebbesen experiments has missed some important earlier results of the optical gratings study.

In this way, in these first interpretations, the exact role of SPs was not clearly assessed, and many questions remained to clarify completely the scattering processes involved in these experiments.

As a consequence, some other explanations had been developed which could compete with the SPs model. For instance, it was suggested that these phenomena could also be described in terms of cavity resonances taking place into the holes [8]. More recently, T. Thio and $\mathrm{H}$. Lezec had also suggested a diffracted evanescent wave model [9].

In addition, some doubts against the SPs hypothesis relied on some experimental results about subwavelength hole arrays made in non-metallic films. Though SPs cannot exist, the transmission pattern was found to be very similar to that obtained with a metallic film [9]. Surprisingly, the very fact that the typical transmission pattern can be observed even in non-metallic systems convinced some authors to fully reject the SPs hypothesis and rather consider models involving nonresonant evanescent waves diffraction. To our knowledge, this point of view is not supported by recent results. In fact, the SPs must be replaced by other kinds of eigenmodes in the nonmetallic cases.

As a consequence, despite alternative theoretical interpretations, a large experimental consensus, many theoretical results, tend to prove that the SPs interpretation was correct in the metallic films cases, and must be extended to a bounded mode interpretation in the most general cases [10-12]. It is only the transposition of the 1960's results about one-dimensional reflection gratings to bidimensional transmission gratings.

Let us clarify this.

\section{Bounded modes and transmission}

Basically, the gratings considered here are simply drilled slabs. In this case, a bounded mode, i.e. an eigenmode of the grating, is an electromagnetic field configuration such that the field is trapped along the slab. The mode can propagate along the slab, but not elsewhere. The amplitude of the field decays then, exponentially from each side of the slab. Typically, two amplitude patterns exist, which correspond to symmetric and antisymmetric modes. As the thickness of the slab increases, in some cases, the modes can refer to pure surface modes (Fig.4), i.e. both slab interfaces are decoupled. Surface plasmons are such modes for instance.

As previously suggested, bounded modes cannot be excited directly by incident light or decay spontaneously into photons. Moreover, the coupling between the outside electromagnetic field and the bounded modes is allowed by the use of rough or corrugated surface, as in the present case. The eigenmodes, and their coupling with outside light, can be described mathematically as follow.

As in quantum mechanics, a scattering problem can be treated in electromagnetism via the use of the scattering matrix formalism ( $S$ matrix). In such a representation, it is assumed that the electromagnetic field can be described by two super- 


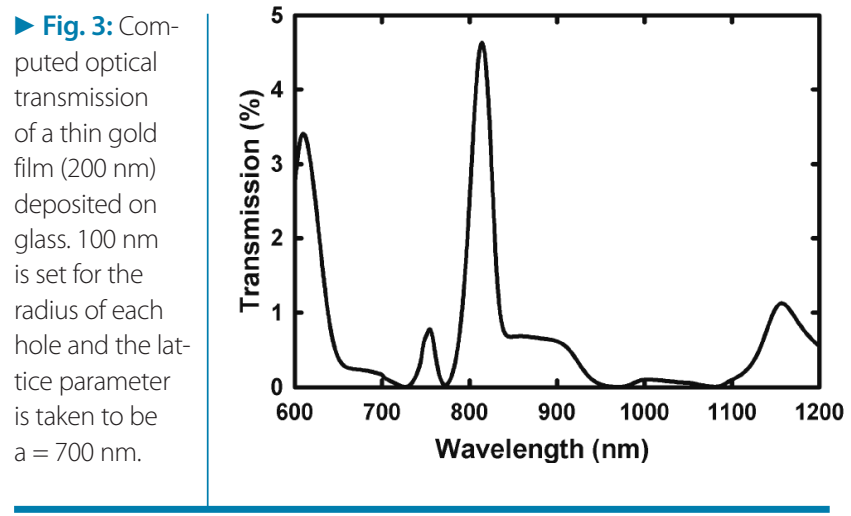

vectors $\left|F_{\text {in }}\right\rangle$ and $\left|F_{\text {scatt }}\right\rangle$ (with the use of "ket" representation), which correspond to the incident electromagnetic field that lit the studied device and to the field scattered by the device. For instance, each component of these vectors corresponds to a specific diffraction order. Then, incident and scattered field are linked via the scattering matrix defined as

$$
\left|F_{\text {scatt }}\right\rangle=S(\lambda)\left|F_{\text {in }}\right\rangle
$$

so that $S(\lambda)$ contains all the physical information about the studied device, such as a diffraction grating. In addition, it can be shown that $S(\lambda)$ can be explicitly defined from the Maxwell's equations. For instance, the simulations in our papers are based on such a method, which combines scattering matrix formalism with a plane wave representation of the fields. This technique provides a computation scheme for the amplitude and polarization ( $s$ or $p$ ) of reflected and transmitted fields in any diffracted order.

A fundamental key to the understanding of diffraction grating phenomenology is to define the eigenmodes of the studied device. Eigenmodes then obey the homogeneous equation

$$
S^{-1}\left(\lambda_{p}\right)\left|F_{\text {eigen }}\right\rangle=0
$$

in such a way that the eigenmodes can also be defined by the poles $\lambda_{p}$ of the scattering matrix. Then, the poles are the solutions of the equation

$$
\operatorname{det}\left\{S^{-1}\left(\lambda_{p}\right)\right\}=0
$$

If we extract the singular part of $S(\lambda)$ corresponding to the eigenmodes of the structure, we can write $S(\lambda)$ in an analytical form as

$$
S(\lambda)=\sum_{p} \frac{R_{p}}{\lambda-\lambda_{p}}+S_{h}(\lambda)
$$

This is a generalized Laurent series, where $R_{p}$ are the residues associated with each pole $\lambda_{p}$. One notes that $\lambda_{p}=\lambda_{p}^{R}+i \lambda_{p}^{I}$ are complex numbers, and that the imaginary part $\lambda_{p}^{I}$ can be linked to lifetimes of the eigenmodes. The divergent behaviour of the fractional terms underlines the resonant character of the eigenmodes (one talks then about the resonant terms of the $S$ matrix). $S_{h}(\lambda)$ is the holomorphic part of $S(\lambda)$ which corresponds to purely non-resonant processes. Ideally, in the vicinity of a specific pole, the holomorphic part can be assumed to be almost constant. Then, as the amplitude $s(\lambda)$ of a specific dif- fraction order is related to an element of the scattering matrix, the intensity $I \propto|s(\lambda)|^{2}$ of an order can be easily derived. One then obtains

$$
I_{F}=\tilde{I}_{0} \frac{\left(\lambda-\lambda_{z}^{R}\right)^{2}+\lambda_{z}^{I^{2}}}{\left(\lambda-\lambda_{p}^{R}\right)^{2}+\lambda_{p}^{I^{2}}}
$$

where $\lambda_{z}=\lambda_{z}^{R}+i \lambda_{z}^{I}$ is the complex zero of $s(\lambda)$ in the vicinity of $\lambda_{p}$ in the complex plan. This function leads to a typical asymmetrical profile, a Fano profile, related to a Fano resonance. A typical Fano profile appears in Fig.5 (black curve). Now, if the holomorphic part is considered as negligible, one gets the following expression of the amplitude

$$
I_{B R}=I_{0} \frac{1}{\left(\lambda-\lambda_{p}^{R}\right)^{2}+\lambda_{p}^{I^{2}}}
$$

which gives a simple Lorentzian profile, related to a BreitWigner resonance (see for instance the red curve of Fig.5). As shown in Fig.5, for a given eigenmode (i.e. $\lambda_{p}$ imposed), the value of $\lambda$ for which $I_{B R}$ is a maximum (i.e. $\lambda=\lambda_{p}^{R}$ ) does not correspond to the location of $I_{F}$ maximum or minimum (i.e. (a) or (b) in Fig.5).

In order to illustrate the physical mechanisms responsible for the behaviour described by the scattering matrix formalism, we represent the corresponding processes involved.

In Fig. 6, circles $A$ and $B$ represent diffracting elements e.g., holes. So an incident homogeneous wave $i$ (in red) diffracts in $A$ and generates a nonhomogeneous diffraction order (blue dashed line). Such an order is coupled with an eigenmode, which is characterized by a complex wavelength $\lambda_{p}$. It then becomes possible to excite this eigenmode, which leads to a feedback reaction on the nonhomogeneous order (one can talk then about a resonant order). This process is related to the resonant term of the scattering matrix. In Fig.5, the red curve is the electromagnetic field amplitude calculated for such a nonhomogeneous resonant order. The resonant diffraction order ...

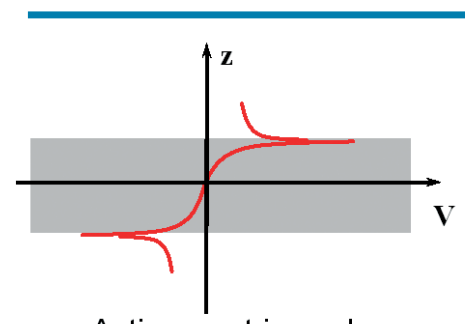

Antisymmetric mode

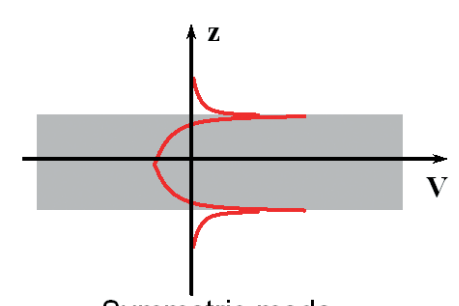

Symmetric mode

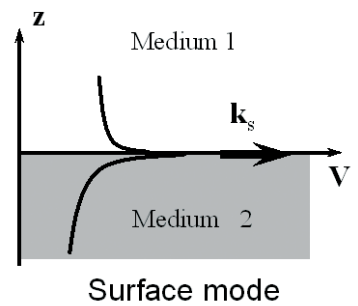

$\Delta$ Fig. 4: Diagrams of slab modes. The electrostatic potential $V$ of modes is plotted as a function of the location in the slab. Above: antisymmetric and symmetric modes. Below: pure surface mode, with each interface decoupled from each other. 


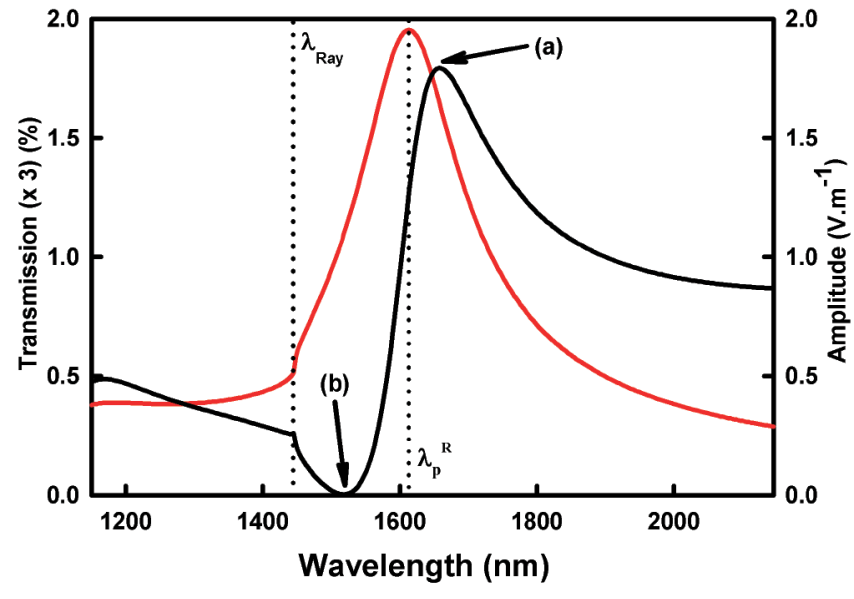

$\Delta$ Fig. 5: Computed optical transmission (black curve) and amplitude of the related resonant order (red curve) of a thin fictitious film (the permittivity is equal to i1) deposited on glass. $500 \mathrm{~nm}$ is set for the radius of each hole and the lattice parameter is taken to be $a=1000 \mathrm{~nm}$. A discontinuity appears at the Rayleigh wavelength $\lambda_{\text {Ray. }}$.

... diffracts then in $B$, and generates a contribution to a homogenous diffraction order, in our case the specular beam. Thus one can ideally expect to observe a resonant profile, i.e. Lorentzian-like, for the amplitude $s_{r}$ of the homogenous specular diffraction order that appears in $B$. Nevertheless, it is also necessary to account for nonresonant diffraction processes related to the holomorphic term $S_{h}(\lambda)$. So the incident wave $i$, generates also a homogeneous order $S_{h}$ (in green), which appears as another contribution to the specular beam. So, as one observes the specular transmitted beam, one observes the sum of two rates, i.e. $s_{r}+s_{h}$. So, the Fano profiles of the transmission (but also of the reflection) result from a superposition of resonant and nonresonant contributions to the observed diffraction order [10-11]. As a result, in Fig.5 one can observe the calculated transmission (black curve) in comparison with the non-homogeneous resonant order amplitude (red curve).

\section{Consequences and observations}

As shown in Fig.3, the observed optical transmission through subwavelength hole arrays, exhibits a set of peaks and dips. Following the previously described approach, we have shown in recent works that the transmission spectrum is better depicted as a series of Fano profiles (see Fig.3 and Fig.5) [10,13,14]. These recognizable line shapes result from the interference of nonresonant transfers with resonant transfers, which involve the eigenmodes film, and evanescent diffraction orders. We could then point out that each transmission peak-dip pair is nothing other than a Fano profile. The appearance of an assymetric Fano line shape does not necessarily locate the peak or the dip at the eigenmode resonance (as shown in Fig.5), contrary to what has been suggested in some earlier works $[7,8]$. However, we have shown that the existence of the eigenmodes is a condition for the presence of the Fano line shapes. It can be noted that recent results by Genet et al confirm this description [11]. As a further outcome of this work it became clear that this kind of transmission spectrum, with its Fano line shapes, could also be obtained in a large context and that the generic concept of eigenmodes must substitute that of SPs only. These results implied two important ideas. First, according to the contributions of resonant and nonresonant processes in the Fano profile, eigenmodes can be associated with wavelengths closer to the peaks or to the dips. Second, it is possible to obtain transmission curves similar to those of metal films, by substituting SPs with guided modes or other types of polaritons. Many examples can be found, including highly refractive materials defining guided modes or ionic crystals in the restrahlen band defining phonon polaritons. For instance, we have reported simulations of a device, which consists of arrays of subwavelength cylindrical holes in a tungsten layer deposited on a glass substrate [13]. Tungsten becomes dielectric on a restricted domain of wavelength in the range $240-920 \mathrm{~nm}$, i.e., the real part of its permittivity becomes positive. So, whereas plasmons cannot exist, we show that the transmission pattern is similar to that obtained in the case of a metallic film. Indeed, it was also shown that in this case, instead of SPs, guided modes are excited [13]. Nevertheless, these modes give less intense fields than those achieved with SPs. In this way the benefit for the transmission is less important than with SPs modes. In the same way, it was also demonstrated that the transmission profile of a chromium film, in the restricted wavelength domain (1112-1292 nm), where the dielectric constant is positive, should involve eigenmodes, which are not SPs or guided modes. In this case, SPs can be substituted by Brewster-Zennek (BZ) modes [14]. Lastly it should be noted that in both cases, these theoretical computations are supported by experimental results.

As a consequence, it appears that bounded modes can act as a mediator of the optical transmission in bidimensional subwavelength hole arrays. Although it appears that the mechanisms involved in these devices have been implicitly known for a long time, it seemed important to us to recall it. Indeed, an important engine of the technological development is also the

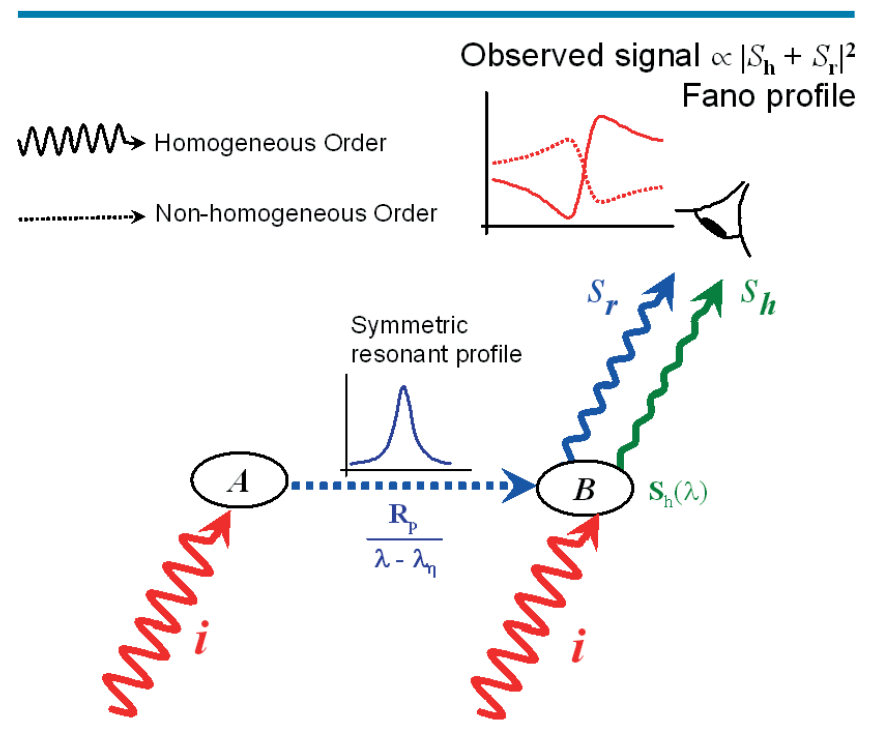

A Fig. 6: Diagrammatic representation of the processes responsible for the behaviour of the transmission properties. 
improvement of our theoretical knowledge of the phenomena with which we are confronted.

\section{About the authors}

Michaël Sarrazin is scientific collaborator at the University of Namur (FUNDP), Belgium. He also teaches physical sciences at the Cours Notre-Dame des Anges in Belfort, France. He received his $\mathrm{PhD}$ in physics from the University of FrancheComté, France (2002). His main research interests are in theoretical study of electrodynamics in various situations and dimensionalities.

Jean-Pol Vigneron is professor of Physics at the University of Namur (FUNDP), Belgium. His main interest lies in the field of surface electrodynamics and photonics, with an emphasize on the inverse engineering of natural photonic nanostructures.

\section{References}

[1] R. W. Wood, Phil. Mag. 4, 396 (1902). R. W. Wood, Phys. Rev. 48, 928 (1935)
[2] Lord Rayleigh, Proc. Roy. Soc. (London) A79, 399 (1907)

[3] U. Fano, Ann. Phys. 32, 393 (1938). U. Fano, Phys. Rev. 124, 1866 (1961)

[4] A. Hessel and A.A. Oliner, Appl. Opt. 4, 1275 (1965)

[5] D. Maystre, M. Nevière, J. Opt. 8, 165 (1977)

[6] M. Nevière, D. Maystre, and P. Vincent, J. Opt. 8, 231 (1977)

[7] T.W. Ebbesen, H.J. Lezec, H.F. Ghaemi, T. Thio, and P.A. Wolff, Nature London 391, 667 (1998)

[8] Q. Cao and Ph. Lalanne, Phys. Rev. Lett. 88, 057403 (2002)

[9] H. J. Lezec and T. Thio, Opt. Express 12, 3629 (2004)

[10] M. Sarrazin, J-P. Vigneron, J-M. Vigoureux, Phys. Rev. B 67, 085415 (2003)

[11] C. Genet, M. P. van Exter, and J. P. Woerdman, Opt. Commun. 225, 331 (2003)

[12] W.L. Barnes, W.A. Murray, J. Dintinger, E. Devaux, and T.W. Ebbesen, Phys. Rev. Lett. 92, 107401 (2004)

[13] M. Sarrazin, J-P. Vigneron, Phys. Rev. E 68, 016603 (2003)

[14] M. Sarrazin, J-P. Vigneron, Phys. Rev. B 71, 075404 (2005) 